\title{
Effects of intracoronary antithrombotics on ventricular function: A comparison of tenecteplase versus abciximab during primary percutaneous intervention in myocardial infarction
}

\author{
Francisco J. Morales-Ponce ${ }^{1}$, Pablo González-Pérez ${ }^{1}$, Sara Blasco-Turrión ${ }^{1} \oplus$, \\ Juan Antonio Sánchez-Brotons ${ }^{1}$, Carmen Collado-Moreno ${ }^{1}$, Pedro Martínez-Romero ${ }^{1}$, \\ Eduardo Martínez-Morentín ${ }^{1}$, Pilar Caro-Mateo ${ }^{2}$ \\ ${ }^{1}$ Department of Cardiology, Puerto Real University Hospital, Cadiz, Spain \\ ${ }^{2}$ Department of Radiology, DADISA Radiological Center, Cadiz, Spain
}

Adjunctive medical therapy during primary percutaneous coronary intervention (PPCI) is based on anticoagulation and antiplatelet drugs. Additionally, the glycoprotein IIb/IIIa inhibitor (GPI) abciximab has been shown to reduce infarct size in some clinical trials [1]. However, the role of intracoronary fibrinolysis has not been well established during PPCI. We sought to explore the hypothesis that a locally administered fibrinolytic could be more effective in dissolving coronary thrombus at the macro and microvasculature than adding a third antiplatelet drug in patients already receiving double antiplatelet therapy, and therefore improve myocardial perfusion and left ventricular function.

The present study involved conducting a phase-III, single-center, prospective, randomized controlled trial, in which patients with acute anterior ST-segment elevation myocardial infarction (STEMI) and a coronary flow-limiting lesion (Thombolysis In Myocardial Infarction [TIMI] flow grade 0-2) in the left anterior descending coronary artery were randomized to receive either intracoronary tenecteplase (one fifth of the usual systemic dose according to weight) or abciximab (an intracoronary dose of $0.25 \mathrm{mg} / \mathrm{kg}$ followed by an intravenous infusion of $0.125 \mathrm{mcg} / \mathrm{kg} / \mathrm{min}$ for $12 \mathrm{~h}$ ). After crossing the culprit lesion with a guidewire, the study medication was infused through the guiding catheter for $3 \mathrm{~min}$. All patients had received oral acetylsalicylic acid (300 mg), clopidogrel $(600 \mathrm{mg})$ and an intravenous bolus of $70 \mathrm{UI} / \mathrm{kg}$ of unfractionated heparin before the procedure. In both groups, PPCI was then performed as usual with implantation of coronary stents as needed. The complete study protocol was described in a previous publication [2].

All patients were scheduled for a contrast echocardiogram before discharge and at 6 months (contrast agent Sonovue, Bracco, The Netherlands), all of them performed with a GE Vivid 7 ultrasound system by the same experienced cardiologist, who was blinded to the study medication, assessing the usual systolic and diastolic ventricular function parameters according to the American Society of Echocardiography. Additionally, all patients were scheduled for a 4-month cardiac-magnetic resonance imaging (MRI) at an external radiological center, for a blinded analysis of infarct size, left ventricular volumes and ejection fraction, as previously described [2]. The study protocol was approved by the local and regional ethics committees. The trial was registered in Eudra CT (https://www.clinicaltrialregister.eu, identifier 2010-022725-16). All patients gave written informed consent to participate in the study.

From August 2012 to April 2016, 102 patients with acute anterior STEMI were screened for enrolment in the study, 26 of which were later excluded due to not meeting the listed criteria. Consequently, 76 patients with acute anterior STEMI were randomized to receive either intracoronary tenecteplase $(n=38)$ or abciximab $(n=38)$ dur-

Address for correspondence: Dr. Sara Blasco-Turrión, Department of Cardiology, Puerto Real University Hospital, Cadiz, Spain, e-mail: sarablasco.cardiologia@gmail.com

Received: 18.06.2020 Accepted: 6.10.2020

This article is available in open access under Creative Common Attribution-Non-Commercial-No Derivatives 4.0 International (CC BY-NC-ND 4.0) license, allowing to download articles and share them with others as long as they credit the authors and the publisher, but without permission to change them in any way or use them commercially. 
Table 1. Echocardiogram and cardiac-magnetic resonance imaging (MRI) results.

\begin{tabular}{|c|c|c|c|}
\hline & i.c. Tenecteplase & i.c. Abciximab & $\mathbf{P}$ \\
\hline 48-hour echocardiogram & $\mathbf{N}=\mathbf{3 8}$ & $\mathbf{N}=\mathbf{3 8}$ & \\
\hline LVEDV index $\left[\mathrm{mL} / \mathrm{m}^{2}\right]$ & 54.2 [44.2-71.7] & $51.5[47.5-62.1]$ & 0.69 \\
\hline LVESV index $\left[\mathrm{mL} / \mathrm{m}^{2}\right]$ & 23.8 [14.9-35.5] & 26.1 [18.1-36.7] & 0.91 \\
\hline LVEF [\%] & $54.0[40.2-66.7]$ & $50.5[43.0-60.7]$ & 0.79 \\
\hline $\mathrm{LAV}$ index $\left[\mathrm{mL} / \mathrm{m}^{2}\right]$ & $27.4[22.6-37.4]$ & $29.7[23.6-33.2]$ & 0.71 \\
\hline Average $\mathrm{e}^{\prime}$ wave $[\mathrm{cm} / \mathrm{s}]$ & $6.2[5.0-7.7]$ & $6.0[4.7-7.0]$ & 0.60 \\
\hline Average $E / e^{\prime}$ ratio & $11.4[9.4-14.9]$ & $14.3[11.2-17.6]$ & 0.04 \\
\hline Average $E / e^{\prime}$ ratio $\geq 13$ & $12(33.3 \%)$ & $21(55.3 \%)$ & 0.05 \\
\hline 4-month angio-MRI & $N=26$ & $N=28$ & \\
\hline LVEDV index $\left[\mathrm{mL} / \mathrm{m}^{2}\right]$ & 85.4 [71.7-99.9] & 88.5 [72.9-104.4] & 0.94 \\
\hline LVESV index $\left[\mathrm{ml} / \mathrm{m}^{2}\right]$ & $38.9[25.0-54.0]$ & $40.1[31.2-55.8]$ & 0.63 \\
\hline LVEF [\%] & $54.0[44.0-62.0]$ & $53.0[44.5-59.5]$ & 0.58 \\
\hline 6-month echocardiogram & $\mathbf{N}=28$ & $\mathbf{N}=35$ & \\
\hline LVEDV index $\left[\mathrm{mL} / \mathrm{m}^{2}\right]$ & 54.8 [45.4-69.2] & $55.4[42.9-67.1]$ & 0.82 \\
\hline LVESV index $\left[\mathrm{mL} / \mathrm{m}^{2}\right]$ & $22.1[13.5-33.9]$ & $23.0[13.3-31.9]$ & 0.87 \\
\hline LVEF [\%] & $59.0[47.0-73.0]$ & 57.5 [43.0-60.7] & 0.74 \\
\hline LV remodeling $>20 \%$ & $6(21.4 \%)$ & $9(26.5 \%)$ & 0.65 \\
\hline LAV index $\left[\mathrm{mL} / \mathrm{m}^{2}\right]$ & $28.3[22.2-40.0]$ & $28.8[24.1-37.1]$ & 0.75 \\
\hline Average $\mathrm{e}^{\prime}$ wave $[\mathrm{cm} / \mathrm{s}]$ & $6.5[5.0-8.0]$ & $6.0[4.0-7.0]$ & 0.23 \\
\hline E/e' ratio & $10.3[8.1-14.6]$ & $13.2[10.7-15.5]$ & 0.03 \\
\hline $\mathrm{E} / \mathrm{e}^{\prime}$ ratio $\geq 13$ & $8(28.6 \%)$ & $18(51.4 \%)$ & 0.05 \\
\hline
\end{tabular}

Variables are expressed as median [interquartile range] or as n (\%); LVEDV — left ventricular end-diastolic volume; LVESV — left ventricular end-systolic volume; LVEF — left ventricular ejection fraction; LAV — left atrial volume; Average $e^{\prime}$ wave — average of the values from the septal and lateral mitral annulus; Average E/e' ratio - ratio of the transmitral Doppler E wave velocity and the composite mean of e'; LV - left ventricular

ing PPCI. Both groups were comparable in demographic, clinical, angiographic and periprocedural characteristics, and there were no significant differences in major cardiovascular or bleeding events between study groups, as published elsewhere [2].

Echocardiogram and cardio-MRI results are displayed in Table 1. Left ventricular volumes and ejection fraction did not significantly differ between the study groups at 6 months, and adverse ventricular remodeling was similar. Only diastolic function, as assessed by the $\mathrm{E} / \mathrm{e}$ ' ratio at 6 months, was significantly better in the tenecteplase group (median 10.3 [interquartile range 8.1-14.6]) in comparison to the abciximab group (median 13.2 [interquartile range 10.7-15.5, respectively, $\mathrm{p}=0.04]$ ). An $\mathrm{E} / \mathrm{e}^{\prime}$ ratio $\geq 13$ was observed in $26.6 \%$ of the patients in the tenecteplase group versus $51.4 \%$ in the abciximab group $(\mathrm{p}=0.05)$ and final infarction size (expressed as weight of infarct mass in grams) had a significant correlation to e' wave value $(\mathrm{r}=-0.49, \mathrm{p}<0.01)$ and $\mathrm{E} / \mathrm{e}$ ' ratio $(\mathrm{r}=0.45, \mathrm{p}=0.04)$.
Primary PCI is the strategy of choice in STEMI, and one of its main goals is saving as much myocardium as possible, since impaired ventricular function after STEMI is an unfavorable prognostic predictor [3]. Adjunctive treatment with GPI during PPCI has shown to improve myocardial perfusion and reduce infarct size in some studies [1]. According to available research, the present study constitutes the first randomized trial comparing intracoronary administration of a fibrinolytic drug and a GPI in patients with STEMI undergoing PPCI. In a recent publication, it was communicated that there were no significant differences in infarct size between both drugs in the study population [2]. In the present manuscript, it is also reported that there were no significant differences in systolic ventricular function as well. There was only a mild trend to a better preservation of diastolic function in the tenecteplase group that could be considered as marginal.

Previous [2] and the present results agree with those previously reported in the recent T-TIME 
study [4], in which intracoronary alteplase worsened the microvascular obstruction and increased intramyocardial hemorrhage when compared to the placebo, essentially in case of prolonged ischemia. Furthermore, these worse results on myocardial reperfusion are not necessarily related to final infarct size, since edema and intramyocardial hemorrhage are usually solved when the 4-month monitoring MRI is performed. This could explain the absence of significant differences in infarct size between the two therapy groups. Additionally, as in the current study, in the T-TIME trial no significant benefits on ventricular function were found [4].

The sample size in this pilot study was arbitrary and was not previously calculated. The relatively small size of infarcted myocardium in the whole study population could have limited the ability to detect significant differences in most systolic and diastolic function parameters. Additionally, comparing tenecteplase to an antiplatelet drug rather than a placebo may have diminished its capacity of showing positive results on the study endpoints. The choice of tenecteplase dose was selected from previous case reports and observational studies, although it could have been insufficient to achieve the desirable antithrombotic effect. Finally, more recent P2Y12 inhibitors (such as ticagrelor or prasugrel) were not used in the present study since their administration is not recommended in addition to a fibrinolytic drug.

In this first clinical randomized trial comparing intracoronary fibrinolysis with a GPI drug during
PPCI, and when combining present results with those recently reported [2], no benefits were found on ventricular function nor infarct size after myocardial infarction when intracoronary fibrinolysis was performed. Therefore, and lacking results from larger studies, the results do not support a systematic use of intracoronary fibrinolysis as adjunctive therapy during PPCI.

\section{Conflict of interest: None declared}

\section{References}

1. Stone GW, Maehara A, Witzenbichler B, et al. Intracoronary abciximab and aspiration thrombectomy in patients with large anterior myocardial infarction: the INFUSE-AMI randomized trial. JAMA. 2012; 307(17): 1817-1826, doi: 10.1001/jama.2012.421, indexed in Pubmed: 22447888.

2. Morales-Ponce FJ, Lozano-Cid FJ, Martinez-Romero P, et al. Intracoronary tenecteplase versus abciximab as adjunctive treatment during primary percutaneous coronary intervention in patients with anterior myocardial infarction. EuroIntervention. 2019; 14(16): 1668-1675, doi: 10.4244/EIJ-D-18-00885, indexed in Pubmed: 30418157.

3. Prastaro M, Pirozzi E, Gaibazzi N, et al. Expert review on the prognostic role of echocardiography after acute myocardial infarction. J Am Soc Echocardiogr. 2017; 30(5): 431-443.e2, doi: 10.1016/j.echo.2017.01.020, indexed in Pubmed: 28477781.

4. McCartney PJ, Maznyczka AM, Eteiba H, et al. Low-Dose alteplase during primary percutaneous coronary intervention according to ischemic time. J Am Coll Cardiol. 2020; 75(12): 1406-1421, doi: 10.1016/j.jacc.2020.01.041, indexed in Pubmed: 32216909 . 